\title{
The propagation of internal undular bores over variable topography
}

\author{
R. Grimshaw and C. Yuan \\ Department of Mathematics, University College London, UK
}

November 26, 2015

\begin{abstract}
In the coastal ocean, large amplitude, horizontally propagating internal wave trains are commonly observed. These are long nonlinear waves and can be modelled by equations of the Korteweg-de Vries type. Typically they occur in regions of variable bottom topography when the variable-coefficient Korteweg-de Vries equation is an appropriate model. Of special interest is the situation when the coefficient of the quadratic nonlinear term changes sign at a certain critical point. This case has been widely studied for a solitary wave, which is extinguished at the critical point and replaced by a train of solitary waves of the opposite polarity to the incident wave, riding on a pedestal of the original polarity. Here we examine the same situation for an undular bore, represented by a modulated periodic wave train. Numerical simulations and some asymptotic analysis based on Whitham modulation equations show that the leading solitary waves in the undular bore are destroyed and replaced by a developing rarefaction wave supporting emerging solitary waves of the opposite polarity. In contrast the rear of the undular bore emerges with the same shape, but with reduced wave amplitudes, a shorter overall length scale and moves more slowly.
\end{abstract}

\section{Variable-coefficient Korteweg-de Vries equation}

Large amplitude internal wave trains are commonly observed in the coastal ocean, see the reviews by Grimshaw (2001), Holloway et al. (2001), Ostrovsky and Stepanyants (2005), Helfrich and Melville (2006), Grimshaw (2007), Grimshaw et al. (2010) and the book by Vlasenko et al. (2005). Since these are long nonlinear waves it is now widely accepted that the basic paradigm for these waves is based on the Korteweg-de Vries (KdV) equation, first derived in this context by

\footnotetext{
${ }^{1}$ Corresponding author: r.grimshaw@ucl.ac.uk, (+44) 2076792856
} 
Benney (1966) and Benjamin (1966) and subsequently by many others, see the aforementioned references. In the usual physical variables to describe internal waves in the coastal ocean the $\mathrm{KdV}$ equation is, see the afore-mentioned references,

$$
A_{t}+c A_{x}+\mu A A_{x}+\lambda A_{x x x}=0 .
$$

Here $A(x, t)$ is the amplitude of the modal function $\phi(z)$, defined by

$$
\begin{aligned}
& \left\{\rho_{0}\left(c-u_{0}\right)^{2} \phi_{z}\right\}_{z}+\rho_{0} N^{2} \phi=0, \quad \text { for }-h<z<0, \\
& \phi=0 \text { at } z=-h, \quad\left(c-u_{0}\right)^{2} \phi_{z}=g \phi \quad \text { at } z=0 .
\end{aligned}
$$

This also serves to define the phase speed $c$. Here $\rho_{0}(z)$ is the background density field, stably stratified so that $\rho N^{2}=-g \rho_{0 z}>0, u_{0}(z)$ is a background horizontal current and $h$ is the undisturbed fluid depth. The coefficients $\mu, \lambda$ are given by

$$
\begin{gathered}
I \mu=3 \int_{-h}^{0} \rho_{0}\left(c-u_{0}\right)^{2} \phi_{z}^{3} d z, \\
I \lambda=\int_{-h}^{0} \rho_{0}\left(c-u_{0}\right)^{2} \phi^{2} d z \\
I=2 \int_{-h}^{0} \rho_{0}\left(c-u_{0}\right) \phi_{z}^{2} d z .
\end{gathered}
$$

It is well-known that the $\mathrm{KdV}$ equation (1) is integrable, and its principal solution is the solitary wave, as the outcome of a localised initial condition is a finite set of rank-ordered solitary waves and some small-amplitude dispersing radiation, see Whitham (1974); Ablowitz and Segur (1981). Here we are concerned with the undular bore solution, which can be found as the outcome of a step initial condition by using the Whitham modulation equations, see Gurevich and Pitaevskii (1974).

When the depth $h$, and background current $u_{0}$ and density $\rho_{0}$ vary slowly in the horizontal direction with $x$, the $\mathrm{KdV}$ equation (1) is replaced by a variable-coefficient $\mathrm{KdV}$ (vKdV) equation. first derived in the general case by Grimshaw (1981), see also Zhou and Grimshaw (1989) and Grimshaw et al. (2007, 2010). It has the same form as (1) with an extra term,

$$
A_{t}+c A_{x}+\frac{c Q_{x}}{2 Q} A+\mu A A_{x}+\lambda A_{x x x}=0, \quad Q=c^{2} I
$$

Here the modal equation depends also on $x$ parametrically, that is $\phi=\phi(z: x), c=c(x)$, and hence the coefficients $\mu, \lambda, Q$ also depend (slowly) on $x$. It is convenient to transform this to the "spatial" evolution form,

$$
\begin{gathered}
X=\int^{x} \frac{d x}{c}-t, \quad T=\int^{x} \frac{d x}{c}, \\
A_{T}+\frac{Q_{T}}{2 Q} A+\nu A A_{X}+\delta A_{X X X}=0, \\
\nu=\frac{\mu}{c}, \quad \delta=\frac{\lambda}{c^{3}} .
\end{gathered}
$$


A further simplification is

$$
U=Q^{1 / 2} A, \quad U_{T}+\frac{\nu}{Q^{1 / 2}} U U_{X}+\delta U_{X X X}=0 .
$$

A final transformation yields the canonical form relevant for a polarity change, that is the coefficient $\alpha$ changes sign,

$$
\begin{gathered}
U_{\tau}+\alpha U U_{X}+U_{X X X}=0, \\
\text { where } \quad \tau=\int^{T} \delta d T, \quad \alpha=\frac{\nu}{\delta Q^{1 / 2}} .
\end{gathered}
$$

The coefficient $\alpha$ varies with $\tau$, that is $\alpha=\alpha(\tau)$ in general. This equation has two important conservation laws

$$
\begin{gathered}
U_{\tau}+\left\{\frac{\alpha U^{2}}{2}+U_{X X}\right\}_{X}=0 \\
\left\{\frac{U^{2}}{2}\right\}_{\tau}+\left\{\frac{\alpha U^{3}}{3}+U U_{X X}-\frac{U_{X}^{2}}{2}\right\}_{X}=0 .
\end{gathered}
$$

corresponding to conservation of mass and wave action flux respectively. The first arises directly from (12) expressed in flux form, while the second follows from multiplying (12) by $U$.

Our main interest here is when there is a change of polarity, that is the quadratic coefficient $\mu$ in the $\mathrm{KdV}$ equation (1) changes sign at a critical point. Since $Q, \lambda \neq 0$ for internal waves, it follows that then $\alpha$ in (12) will likewise change sign. This typically occurs when the pycnocline, a thin layer where the density gradient is very strong, is near the surface in deep water, but near the bottom in shallow water. For mode one waves, it is readily shown that $\mu$ is then negative in deep water, but positive in shallow water, and so changes sign as the waves propagate shoreward. The implication is that solitary waves are depression waves in deep water, but elevation waves in shallow water. The behaviour of a solitary wave as it passes through this critical point is now well understood, see the reviews by Grimshaw (2007); Grimshaw et al. (2007, 2010). As a depression solitary wave approaches a critical point, its amplitude decreases but at the same time a trailing shelf is generated, which grows in amplitude as the critical point is approached. The combination passes through the critical point and then generates a depression rarefaction wave on which rides an undular bore of elevation waves. The corresponding theory for a periodic wave train has only recently been developed by Grimshaw (2015) and in contrast, the waves pass through the critical point with only a very small change in amplitude but with a polarity reversal. In this paper we examine how an undular bore behaves as it passes through a critical point, noting that the leading waves in the undular bore are solitary waves while the waves in the rear of the bore are periodic waves. Hence there is an expectation that on passage through the critical point, the front of the undular bore will behave similarly to how a solitary wave behaves, while the rear of the undular bore will behave similarly to how a periodic wave behaves.

In section 2 we present the Whitham modulation equations for a modulated periodic travelling wave, and describe briefly how these may be used when waves propagate in a region where $\alpha=\alpha(\tau)$ varies. Then in section 3 we discuss in detail how either a solitary wave, or a periodic wave train, or an undular bore behave when there is a change of polarity. The analysis is based on the Whitham modulation equations supplemented by some numerical simulations. We conclude in section 4. 


\section{Whitham modulation equations}

When the coefficient $\alpha$ in (12) is a constant the $\mathrm{KdV}$ equation supports a periodic travelling wave, $U(X-V \tau)$, which satisfies the ordinary differential equation,

$$
U_{X}^{2}=-\frac{\alpha U^{3}}{3}+V U^{2}+C_{1} U+C_{2}
$$

where $C_{1,2}$ are constants of integration. This has the well-known cnoidal wave solution

$$
\begin{gathered}
U=a\left\{b(m)+\mathrm{cn}^{2}(\gamma \theta ; m)\right\}+d, \quad \theta=k(X-V \tau), \\
\text { where } \alpha a=12 m \gamma^{2} k^{2} . \quad b(m)=\frac{1-m}{m}-\frac{E(m)}{m K(m)}, \\
V-\alpha d=\frac{\alpha a}{3}\left\{\frac{2-m}{m}-\frac{3 E(m)}{m K(m)}\right\}=4 \gamma^{2} k^{2}\left\{2-m-\frac{3 E(m)}{K(m)}\right\} .
\end{gathered}
$$

Here $c n(x ; m)$ is the Jacobian elliptic function of modulus $m, 0<m<1$, and $K(m)$ and $E(m)$ are the elliptic integrals of the first and second kind,

$$
\begin{gathered}
\operatorname{cn}(x ; m)=\cos (\phi), \quad x=\int_{0}^{\phi} \frac{d \phi^{\prime}}{\left(1-m \sin ^{2} \phi^{\prime}\right)^{1 / 2}}, \quad 0 \leq \phi \leq \frac{\pi}{2}, \\
K(m)=\int_{0}^{\pi / 2} \frac{d \phi}{\left(1-m \sin ^{2} \phi\right)^{1 / 2}}, \quad E(m)=\int_{0}^{\pi / 2}\left(1-m \sin ^{2} \phi\right)^{1 / 2} d \phi .
\end{gathered}
$$

The expression (17) has period $2 \pi$ in $\theta$ so that $\gamma=K(m) / \pi$, while the spatial period is $2 \pi / k$ The (trough-to-crest) amplitude is $a$ and the mean value over one period is $d$. It is a three-parameter family with parameters $k, m, d$ say. As the modulus $m \rightarrow 1$, this becomes a solitary wave, since then $b \rightarrow 0$ and $\operatorname{cn}(x) \rightarrow \operatorname{sech}(x)$, while $\gamma \rightarrow \infty, k \rightarrow 0$ with $\gamma k=\Gamma$ fixed. As $m \rightarrow 0, b \rightarrow-1 / 2$, $\gamma \rightarrow 1 / 2, \operatorname{cn}(x) \rightarrow \cos (x)$, and it reduces to a sinusoidal wave $(a / 2) \cos (\theta)$ of small amplitude

$a \sim m$ and wavenumber $k$. The integration constants $C_{1,2}$ can also be expressed in terms of $k, m, d$ but the explicit expressions are not needed here.

The Whitham modulation theory allows this cnoidal wave to vary slowly with $\tau, X$, that is the the wavenumber $k$, modulus $m$ and mean level $d$ vary slowly with $\tau, X$. The Whitham modulation equations describing this variation can be obtained by averaging conservation laws, the original Whitham method, see Whitham $(1965,1974)$ or by exploiting the integrability of the constantcoefficient KdV equation, see Kamchatnov (2000) for instance. Because here we are concerned with the case when $\alpha=\alpha(\tau)$ varies slowly with $\tau$, and so the variable-coefficient $\mathrm{KdV}$ equation (12) is not integrable, we will use the original Whitham method, readily adapted to this present case. A similar strategy was used by Myint and Grimshaw (1995) for a frictionally perturbed $\mathrm{KdV}$ equation. An alternative method developed by Kamchatnov (2004) for a perturbed KdV equation is not available here because to use it one must make a change of variable in (12) $\tilde{U}=\alpha U$ to generate a $\mathrm{KdV}$ equation for $\tilde{U}$ with a perturbation term of the form $\alpha_{\tau} \tilde{U} / \alpha$. But as our main concern is with the situation when $\alpha$ passes through zero, this approach cannot be used here.

As three modulation equations are needed, we supplement $(14,15)$ with the equation for conservation of waves,

$$
k_{\tau}+(k V)_{X}=0 .
$$


The remaining two modulation equations are obtained by inserting the cnoidal wave solution into the conservation laws $(14,15)$ and averaging over the phase $\theta$. The outcomes are

$$
\begin{gathered}
d_{\tau}+\alpha M_{X}=0, \quad M=<\frac{U^{2}}{2}>, \\
M_{\tau}+P_{X}=0, \quad P=<\frac{\alpha U^{3}}{3}-\frac{3 U_{X}^{2}}{2}>,
\end{gathered}
$$

where the $\langle\cdots\rangle$ denotes a $2 \pi$-average over $\theta$. The expression $M$ is given by

$$
\begin{aligned}
M= & \frac{d^{2}}{2}+\frac{a^{2}}{2}\left\{C_{4}-b^{2}\right\}, \\
C_{4}= & \frac{1}{3 m^{2} K(m)}\left\{3 m^{2} K(m)-5 m K(m)+4 m E(m)+2 K(m)-2 E(m)\right\}, \\
P= & \alpha\left\{-\frac{2 d^{3}}{3}+2 d M+a^{3}\left\{-\frac{2 b^{3}}{3}+\frac{(1-m) b}{2 m}+\left(b+\frac{1-2 m}{2 m}\right) C_{4}+\frac{5}{6} C_{6}\right\},\right. \\
C_{6}= & \frac{1}{15 m^{3} K(m)}\left\{15 m^{3} K(m)-34 m^{2} K(m)+23 m^{2} E(m)\right. \\
& \quad+27 m K(m)-23 m E(m)-8 K(m)+8 E(m)\} .
\end{aligned}
$$

Here the notation $C_{4}, C_{6}$ denote $<c n^{4}>,<c n^{6}>$ respectively, and like $b=-C_{2}=-<c n^{2}>$ depend on the modulus $m$ only.

It is useful to note that in the solitary wave limit $m \rightarrow 1$ and then $b \sim-1 / K(m), C_{4} \sim 2 / 3 K(m)$, and $C_{6} \sim 8 / 15 K(m)$. To leading order $M \sim d^{2} / 2$ and $P \sim \alpha d^{3} / 3$ and then both equations (23, 24) reduce to the same equation for $d$ alone,

$$
d_{\tau}+\alpha d d_{X}=0
$$

and so $d$ can be regarded as a known quantity. In more detail, the cnoidal wave expression (17) becomes

$$
\left.U=a \operatorname{sech}^{2}(\gamma \theta ; m)\right\}+d, \quad \theta=k(X-V \tau), \quad V-\alpha d=\frac{\alpha a}{3}=12 \gamma^{2} k^{2}
$$

with two parameters to be determined. The equation for conservation of waves (22) provides one equation for $k$ and the second equation is

$$
\left\{\frac{a^{2}}{k \gamma}\right\}_{\tau}+V\left\{\frac{a^{2}}{k \gamma}\right\}_{X}+\frac{a^{2}}{k \gamma} \alpha d_{X}=0,
$$

This can be obtained by a more careful consideration of the limit $m \rightarrow 1$ in the modulation equations $(23,24)$ by retaining the terms in $1 / K(m)$, or more directly by averaging the wave action conservation law (15) directly for a solitary wave, see Grimshaw (1979) and the discussion in El et al. (2012). The pair $(22,29)$ form a nonlinear hyperbolic system for a solitary wave train, and can be solved explicitly. Indeed, using the expressions in (28), (30) can be written as

$$
\mathcal{A}_{\tau}+\left(\alpha d+\frac{\alpha a}{3}\right) \mathcal{A}_{X}+\mathcal{A} \alpha d_{X}=0, \quad \mathcal{A}=\left\{\frac{a^{3}}{\alpha}\right\}^{1 / 2} .
$$


This is an equation for the amplitude $a$ alone, and is readily solved using characteristics. Then, with $a$ and hence $V$ known, the wavenumber $k$ can be found from (22) which is a linear hyperbolic equation for $k$.

In the general case for a modulated periodic wave train the set $(22,23,24)$ form three nonlinear hyperbolic equations for $k, m, d$. For the case of a periodic wave modulated only in $\tau$, we obtain the elementary solution that $k, d, M$ are constants. But for an undular bore, the cnoidal wave parameters will vary with both $\tau, X$ and so this elementary solution is not available. In that case when $\alpha$ is a constant Whitham (1974) (see also Kamchatnov (2000) for a general approach) showed that these equations can be cast into Riemann form. This was achieved by noting that the ordinary differential equation (16) can be expressed as

$$
U_{X}^{2}=-\frac{\alpha}{3}\left(U-r_{1}\right)\left(U-r_{2}\right)\left(U-r_{3}\right)
$$

where $C_{1,2}$ are integration "constants" which can be expressed in terms of $k, m, d$. When $\alpha$ is a constant, then $r_{1,2,3}$ are the Riemann variables enabling the nonlinear hyperbolic system to be cast into a diagonal and hence integrable form. However, this reduction is not available here when $\alpha=\alpha(\tau)$ as then the transformation from $k, m, d$ to $r_{1,2,3}$ contains an explicit dependence on $\tau$, which will generate extra terms and prevent a purely diagonal form being obtained. Hence here instead we shall work directly with the set $k, m, d$.

The case of a periodic wave modulated only in $\tau$ was studied by Grimshaw $(2007,2015)$ and is reproduced briefly here. In this case we can seek a solution of the set $(22,23,24)$ which depends on $\tau$ only, and we see that then $k, d, M$ are constants. Using the expressions in (18) these yield the formula

$$
F(m) \equiv K(m)^{2}\left\{(4-2 m) E(m) K(m)-3 E(m)^{2}-(1-m) K(m)^{2}\right\}=C \alpha^{2},
$$

where $C$ is a constant proportional to $M / k$ and is determined by the initial modulus $m_{0}$ at $\tau=\tau_{0}$. The expression (32) can be written in the normalised form

$$
\frac{F(m)}{F\left(m_{0}\right)}=\frac{\alpha^{2}}{\alpha_{0}^{2}},
$$

where $\alpha_{0}=\alpha\left(\tau_{0}\right)$. A plot of the normalised $F(m)$ (the left-hand side of (33) when $m_{0}=$ 0.98 (a strongly nonlinear wave) and $m_{0}=0.85$ is shown in figure 1 . We see that as $|\alpha|$ increases/decreases, then so does the modulus $m$. Then substitution into the expression (18) for the wave amplitude yields

$$
\frac{a}{a_{0}}=\frac{m K(m)^{2}}{m_{0} K\left(m_{0}\right)^{2}} \sqrt{\frac{F\left(m_{0}\right)}{F(m)}},
$$

This expresses the normalized amplitude in terms of the modulus $m$, which in turn varies with $\alpha$ according to (33). A plot of (34) is shown in figure 2 when $m_{0}=0.98,0.85$, and these show that as $|\alpha|$ increases/decreases then so does the amplitude. In both cases there is a surprisingly small variation of the amplitude $a$ until $m \rightarrow 1$.

Formally the solitary wave can be found by taking the limit $m \rightarrow 1$ in (32), or more directly from the solitary wave train equations $(22,30)$. When the modulation is only in $\tau$ these give the 




Figure 1: A plot of the normalised $F N=F(m) / F\left(m_{0}\right)(33)$ versus $m$ when $m_{0}=0.98$ (lower curve, black, solid) and $m_{0}=0.85$ (upper curve, blue, dash).

result that $k, \mathcal{A}$ are constants, the latter yielding the adiabatic expression $a^{3} \propto \alpha$. However, this requires taking the limit $k \rightarrow 0$ which is inconsistent with the assumption that the width scale of the wave should be much less than that of the variable medium defined here by the variation in $\alpha$. Instead, as is now well-known, see the reviews by Grimshaw (2007); Grimshaw et al. (2007, 2010), a multi-scale asymptotic expansion for the solitary wave should be used, which confirms the adiabatic expression $a^{3} \propto \alpha$ due to conservation of wave action flux, but also reveals that the deforming solitary wave is accompanied by a trailing shelf needed to conserve the total mass. The essential difference between the solitary wave and the periodic wave is that in the latter, the mass is represented by the independent parameter $d$ which is a constant, whereas the solitary wave has only one parameter, say the amplitude $a$ whose variation is already determined, while the solitary wave mass is $2 a / \gamma$ and is not constant.

When $\alpha$ is a constant, a representation of an undular bore can be found by seeking a similarity solution of the Whitham modulation equations $(22,23,24)$, where the modulus $m$ depends only on $X / \alpha U_{0} \tau$, see Gurevich and Pitaevskii (1974); Whitham (1974); Fornberg and Whitham (1978) and the review by El (2007). This describes an expanding wave train connecting a zero level at the front where $m \rightarrow 1$ to a mean level $U_{0}\left(\alpha U_{0}>0\right)$ at the rear where $m \rightarrow 0$. At the front the leading wave is a solitary wave of amplitude $2 U_{0}$ and at the rear the waves are linear sinusoidal waves. However, in a variable medium, when as here $\alpha=\alpha(\tau)$, although the Whitham modulation equations are again available, it would seem that no such simple wave solution is available to describe the evolution of an undular bore. A recent study by El et al. (2012) of a water wave undular bore propagating up a slope (that is $|\alpha|$ increases) demonstrated that the deformation at the front of the undular bore is essentially non-adiabatic. Briefly, it is argued that if the undular bore retains its structure as a single-phase wave train, then the jump $U_{0}$ is preserved, and so then the leading solitary wave would have a constant amplitude $2 U_{0}$. But this is inconsistent with the result that the leading solitary wave amplitude should behave 




Figure 2: A plot of the normalised amplitude $A M P=a / a_{0}(34)$ versus $m$ when $m_{0}=0.98$ (lower curve, black, solid) and $m_{0}=0.85$ (upper curve, blue, dash).

as $|\alpha|^{1 / 3}$. The resolution of this inconsistency is the formation of a solitary wave train ahead of the undular bore. This solitary wave train can be described by a reduced set of two modulation equations, and there is a region where the rear of the solitary wave train interacts with the undular bore, forming a two-phase wave interaction. The rear part of the undular bore retains its shape, where pertinently we note that in the similarity solution for the constant coefficient case, $\alpha$ occurs only in the variable $x / \alpha U_{0} \tau$, implying that the effect of a variable $\alpha$ is in some sense equivalent to adjusting the time scale.

\section{Change of polarity for an undular bore}

Our main interest here is in the case when the nonlinear coefficient $\alpha$ passes through zero at a critical point, say $\tau=0$. Without loss of generality we suppose that $\alpha<0,>0$ according as $\tau<0,>0$ corresponding to the usual oceanic situation when internal solitary waves propagate shorewards. Then in $\tau<0$ the KdV equation (12) supports solitary waves and periodic waves of depression, that is $a<0$ in (18), but in $\tau>0$ this polarity is reversed, and instead $a>0$.

To examine this situation we performed several simulations of the vKdV equation (12) using a pseudo-spectral code. Here the pseudo-spectral method based on a Fourier interpolant is implemented to solve equation (12), see Boyd (2001); Weideman and Reddy (2000). In general, equation (12) is stiff, a common feature when solving partial differential equations with spectral methods, and so the numerical solution requires special treatment if accurate solutions are to be efficiently found. We used the recently developed time scheme, Exponential Time Differencing (ETD), which was first proposed by Cox and Matthews (2002), but as partly recognised by the originators, this method as originally proposed encounters certain problems associated with eigenvalues equal to or close to zero, especially when the coefficient matrix in the numerical 
scheme is not diagonal. If these problems are not addressed, ETD schemes prove unsuccessful for some applications. Kassam and Trefethen (2005) claims that this can be addressed by making use of ideas of complex analysis. In this paper, we use ETD based on fourth-order Runge-Kutta time-stepping, which is called ETDRK4 schemes in Kassam and Trefethen (2005).

In the simulations reported here the coefficient $\alpha$ is given by

$$
\alpha=\tanh (K \tau)
$$

where $K$ is chosen so that $\alpha$ varies smoothly and slowly from -1 at $\tau=-\tau_{0}$ to +1 at $\tau=\tau_{0}$. The initial condition is $U_{i c}=U\left(X, \tau=-\tau_{0}\right)$ at $\tau=-\tau_{0}$ where $\alpha=-1$ are either (1) a solitary wave, (2) a periodic wave or (3) an undular bore.

$$
\begin{aligned}
& \text { (1): } \quad U_{i c}=a_{0} \operatorname{sech}^{2}\left(\gamma_{0} X\right), \quad a_{0}=-12 \gamma_{0}^{2}, \\
& \text { (2): } U_{i c}=\operatorname{ENV}(X) a_{0}\left\{b+\operatorname{cn}^{2}\left(\gamma_{0} X ; m\right)\right\}, a_{0}=-12 m \gamma_{0}^{2} \cdot b=\frac{1-m}{m}-\frac{E(m)}{m K(m)}, \\
& (3): \quad U_{i c}=U_{0} \operatorname{ENV}(X) .
\end{aligned}
$$

In case (1) the evolving solitary wave has a time scale of $\left(\gamma_{0} V\right)^{-1}$ where the speed $V=4 \gamma_{0}^{2}$, and so to be slowly varying we choose $K \ll \gamma_{0} V=4 \gamma_{0}^{3}$. In case (2) the periodic cnoidal wave (17) is enclosed inside an envelope $E N V(X)$, defined to be positive for all $X$, with a maximum of 1 at $X=0$ and a width much greater than $2 K(m) / \gamma_{0}$, the spatial period of the cnoidal wave. Typically we choose $m$ to be quite large, say $m=0.95$ and then choose $E N V(X)$ accordingly A suitable choice is

$$
E N V(X)=\frac{1}{2}\{\tanh \Gamma(X+L)-\tanh \Gamma(X-L)\}
$$

Here $L \gg K(m) / \gamma_{0}$ and $\Gamma<\gamma_{0}$, say $\Gamma=0.5 \gamma_{0}$. Again the temporal variation of $\alpha$ should be much slower than that of the individual waves, so that, since $m \approx 1$, we choose $K \ll 4 \gamma_{0}^{3}$. In case (3) $E N V(X)$ is chosen to be very close to a box of height $a$, and of very long length $2 L$, that is, choose $K^{-1} \ll 1 \ll 2 L$. With $\alpha<0, U_{0}<0$, the front end of the box is then expected to generate an undular bore before there is a significant change in $\alpha$. Since the leading wave in the undular bore is a solitary wave of amplitude $2 U_{0}$ we choose $K$ so that $K \ll 4 \sigma^{3}$ where here $U_{0}=-6 \sigma^{2}$. The rear end of the box will generate a rarefaction wave.

The behaviour of a solitary wave as it passes through this critical point is now well understood, see the reviews by Grimshaw (2007); Grimshaw et al. (2007, 2010). As the solitary wave approaches the critical point, its amplitude decreases as $a=a_{0}|\alpha|^{1 / 3}$ but at the same time the amplitude of the trailing shelf grows as $|\alpha|^{-8 / 3}$. Close to the critical point, when the solitary wave and the trailing shelf have comparable amplitudes, the adiabatic behaviour breaks down. The whole structure passes through the critical point and in $\tau>0$ generates a depression rarefaction wave connected to the original zero level by an undular bore of elevation waves. The waves in the undular bore have a shorter length scale than the rarefaction wave, giving the appearance of a wave train of elevation solitary waves riding on a depression pedestal. In this region where $\alpha>0$, the solitary wave train equations $(27,30)$ have a similarity solution

$$
d=\frac{X-X_{0}}{\eta}, \quad \eta=\int_{0}^{\tau} \alpha\left(\tau^{\prime}\right) d \tau^{\prime}, \quad X<X_{0}
$$




$$
\mathcal{A}=\frac{1}{\eta}\left\{-\frac{3\left(X-X_{0}\right)}{\eta \xi}\right\}^{3 / 2}, \quad \xi=\int_{\eta}^{\infty} \frac{\alpha\left(\eta^{\prime}\right)^{1 / 3} d \eta^{\prime}}{\left(\eta^{\prime}\right)^{5 / 3}}, \quad a=\alpha^{1 / 3} \mathcal{A}^{2 / 3} .
$$

Note that the $X_{0}$ is not known, and its determination requires a detailed matching with the solution at the critical point, beyond the scope of this present article. Also the rarefaction wave (40) can only extend to a point $X-X_{0}=-L_{r}(\eta)$ where $L_{r}(\eta)$ is likewise undetermined. But the mass of the rarefaction wave is then $-L_{r}^{2}(\eta) / 2 \eta$ and this can be approximately equated to the initial solitary wave mass $M_{0}=2 a_{0} / \gamma_{0}=-24\left(\left|a_{0} / 12\right|\right)^{1 / 2}(36)$, thus giving an upper estimate for $L_{r}(\eta)$. The expression (41) for the solitary wave amplitude $a$ holds on the domain $-L_{r}(\eta)<X-X_{0}<-L_{s}(\eta)$ where the upper bound $L_{s}(\eta)$ determines the amplitude of the leading solitary wave, that is $a_{s}=3 \alpha^{1 / 3} L_{s} / \eta^{5 / 3} \xi$. The value of $a_{s}$ and hence $L_{s}$ is undetermined and requires matching with the solution at the critical point, beyond the scope of this present article. But an approximate estimate might be based on the assumption that since the emerging solitary wave train is the leading edge of an undular bore resolving the jump at the rear of the rarefaction wave, and then $a_{s}=2 L_{r} / \eta$, where in turn $L_{r}$ is estimated from conservation of mass, as above. When $\alpha$ is a constant, for instance when $\tau \geq \tau_{0}, \eta \sim \alpha \tau, \xi=3 \alpha^{1 / 3} / 2 \eta^{2 / 3}$ and then $a \sim-2\left(X-X_{0}\right) / \alpha \tau$. A numerical simulation of the vKdV equation (12) with the initial condition (36) is shown in figure 3 . The outcome shows qualitative agreement with all the above features. For the parameters in this simulation $M_{0}=-2.2, L_{r} \approx 2.1 \eta^{1 / 2}$ and then $a_{s} \approx 4.2 \eta^{-1 / 2}$; at $\tau=\tau_{0}=5000, \eta=4307$ (40), and then $a_{s} \approx 0.064$, in good agreement with the numerical simulation.
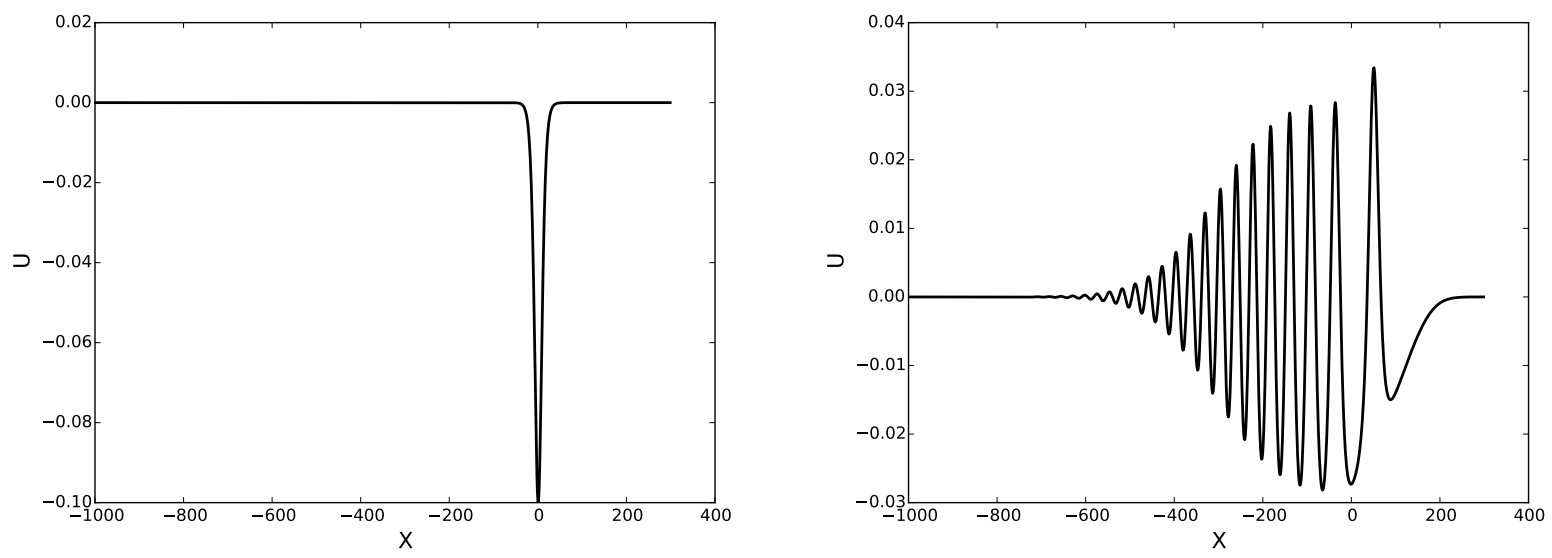

Figure 3: A simulation of the $\mathrm{vKdV}$ equation (12) when $\alpha(\tau)$ varies from -1 to 1 as specified by (35) for the solitary wave initial condition (36) with $a_{0}=-0.1$. the left panel is at $\alpha=-1$, the right panel is at $\alpha=1$, and $\tau_{0}=5000, K=0.001$.

In contrast, the corresponding theory for a periodic wave train has only recently been developed, see Grimshaw (2015). The periodic wave train deforms adiabatically according to the expression (32). As $\alpha \rightarrow 0$ it can be shown that $m \sim \alpha$ and so $m \rightarrow 0$, see figure 1. But from (34) it can be shown that the amplitude $a$ tends to a finite value, see figure 2 . The wave train then passes through the critical point as a linear wave of finite amplitude, but in $\tau>0$ reverses polarity according to (18), this being achieved by a phase change in the linear wave around $\tau=0$. A 
numerical simulation of the $\mathrm{vKdV}$ equation (12) with the initial condition (37) is shown in figure 4 , and there is very good agreement with the asymptotic theory.
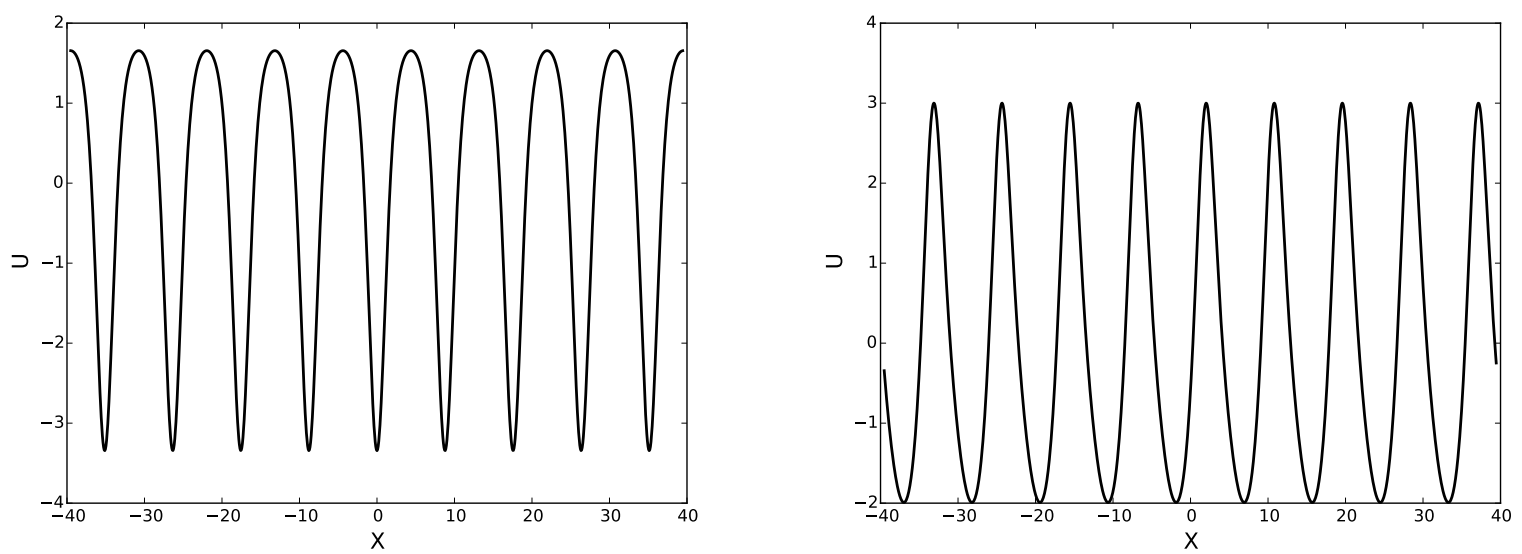

Figure 4: A simulation of the vKdV equation (12) when $\alpha(\tau)$ varies from -1 to 1 as specified by (35) for the cnoidal wave initial condition (37) with modulus $m=0.95$ and $a_{0}=-5$. The left panel is at $\alpha=-1$, the right panel is at $\alpha=1$, and $\tau_{0}=9, K=1$.

These two contrasting scenarios raise the issue of how an undular bore will behave as it passes through a critical point. The leading waves are close to solitary waves, and might be expected, as in the study by El et al. (2012) to form a solitary wave train ahead of the undular bore. But here each solitary wave in that wave train is expected to decrease in amplitude and after passage through the critical point to be replaced by a train of elevation waves, riding on a depression pedestal formed by a depression rarefaction wave. On the other hand, the trailing waves are almost linear periodic waves and might be expected to pass through the critical point as linear periodic waves of finite amplitude, with a reversed polarity as $\alpha$ increases. In the lack of a complete analytical theory obtained from the Whitham modulation equations $(22,23,24)$, we have resorted here to numerical simulations of the vKdV equation (12). A typical example is shown in figure 5 where $U_{0}=-12$ and we set $K=5$, whereas $\sigma=\sqrt{2}$ and so $K<4 \sigma^{3}=11.31$. Simulations with a smaller value of $K$ are similar, but allow the undular bore to develop a finer structure before reaching the critical point, see figure 7. Comparison of the initial structure at $\alpha=-1$ with that at $\alpha=0$ and finally at $\alpha=1$ indicate that the leading solitary waves decrease in amplitude and then after the critical point, there is an indication that a depression rarefaction wave is forming at the front of the undular bore, with elevation solitary waves riding on this pedestal. In contrast the rear of the undular bore retains its shape and passes through the critical point. Subsequent evolution into the region where $\alpha=1$ will lead to a dispersing nonlinear wave train, since the whole structure is negative in a region where $\alpha>0$. In particular note that the decrease in the undular bore amplitude indicates non-adiabatic behaviour, as it was shown by El et al. (2012) that if an undular bore were to deform adiabatically as a single-phase structure, then the jump across the bore is preserved.

In more detail, for comparison figure 6 shows a simulation with the same initial condition, but with $\alpha=-1$ constant. The leading edge now develops as an undular bore. The top panels of figures 5,6 when $\alpha=-1$ in figure 5 are identical. In the middle panel when $\alpha=0$ in figure 5 
we see that the effect of variable $\alpha$ has caused the leading solitary wave to decrease markedly in amplitude and consequently slow down; the remainder of the undular bore has retained its shape, with an overall slight decrease in the wave amplitudes. In the region where $\tau>0, \alpha>0$, the leading part consists of a depression rarefaction wave and a solitary wave train riding on it. This may be described by the same asymptotic solutions $(40,41)$ developed above for a single solitary wave, except importantly that the variables $X_{0}, L_{r}, a_{s}$ are now different although again determined by matching with the solution near the critical point. This is beyond the scope of the present article, but as a rather rough estimate, we again equate the mass $-L_{r}^{2}(\eta) / 2 \eta$ of the rarefaction wave to $M_{0}$ where now $M_{0}$ is the mass carried by the leading solitary wave in the developing undular bore; as this has an amplitude $2 U_{0}, M_{0}=-24\left(\left|U_{0} / 6\right|\right)^{1 / 2}$. Then we again estimate that $a_{s}=2 L_{r} / \eta$. For the simulation shown in figure $5, M_{0}=-33.9, L_{r} \approx 5.8 \eta^{1 / 2}$ and then $a_{s} \approx 11.6 \eta^{-1 / 2}$; at $\tau=\tau_{0}=4, \eta=2.86(40)$, and then $a_{s} \approx 6.9$, in rather good agreement with the numerical simulation.

On the other hand the rear of the undular bore would seem to be changing adiabatically. Hence we examine the structure of the modulation equations as $\alpha \rightarrow 0$. Assuming that in this limit the amplitude remains finite, then the expression $\alpha a=12 m \gamma^{2} k^{2}$ in (17) indicates that the modulus must decrease with $m \sim|\alpha|$; this is the case for the periodic wave train discussed above. Then, as $\alpha \rightarrow 0$, it follows that $M$ remains finite but $P \rightarrow 0$, see $(25,26)$. The modulation equations $(23,24)$ then imply that $M, d$ become steady, that is $M_{\tau}, d_{\tau} \rightarrow 0$. In this limit there will remain an $X$-dependence, and figure 5 shows that $a$ has a straight-line dependence on $X$ as in the initial undular bore. Otherwise, the situation is similar to that for a periodic wave train, and the rear of the undular bore will pass through the critical point as a linear wave. Essentially, as $\alpha \rightarrow 0$ the vKdV equation (12) becomes a linear equation with constant coefficients and can support linear periodic waves, but this cannot be the case for the leading solitary waves which require a balance between the nonlinear and dispersive terms even when $\alpha \rightarrow 0$.

\section{Discussion}

Our focus in this paper is on how an undular bore behaves when it passes through a change of polarity. The main outcome is in the numerical simulation shown in figure 5 where the parameters have been chosen to be typical of those which might occur in the coastal ocean. The front of the undular bore is a depression solitary wave whose amplitude decreases as the critical point is approached and emerges after the critical point as a depression rarefaction wave with several elevation solitary waves emerging from this pedestal. The rear of the undular bore retains its shape, but the wave amplitude decreases and the bore moves more slowly. The deformation of the undular bore is a non-adiabatic process due mainly to the inconsistency between the preservation of the jump and hence the leading solitary wave amplitude if it were adiabatic, and the adiabatic change in the amplitude of a solitary wave as $\alpha$ varies, see El et al. (2012). Consequently, the Whitham modulation equations $(22,23,24)$ cannot be used quantitatively here, even if explicit solutions could be found, but nevertheless do provide some useful qualitative insights. In the case studied by El et al. (2012), $|\alpha|$ increased, and consequently the solitary waves at the front of the undular bore grow in amplitude and are emitted ahead of the undular bore as a solitary wave train satisfying (30); the rear of this solitary wave train interacts with the undular bore forming a two-phase wave interaction region. In contrast, in the present study, $|\alpha|$ initially decreases and so the solitary waves at the front of the undular bore are immediately 
absorbed into the undular bore in a two-phase interaction region. But after passage through the critical point, $|\alpha|$ increases, and the leading solitary waves move ahead but are now riding on a negative pedestal which has the form of depression rarefaction wave. This last feature would seem to be adiabatic and can be modelled the solitary wave train reduction $(27,30)$ of the full Whitham modulation equations.

In the internal wave context, quite often a cubic nonlinear term is added to the KdV equation (1), and hence also to the vKdV equation (12), especially when there is a change of polarity. The consequences for a solitary wave have been examined in detail, see the reviews by Grimshaw (2007); Grimshaw et al. (2010), and depend inter alia on the magnitude and sign of the coefficient of the cubic term. However, the analogous theory for an undular bore in such an extended KdV equation has yet to be developed, and indeed would appear to be much more complicated. In particular only recently has the Whitham modulation theory for an undular bore in the constantcoefficient extended KdV equation been developed by Kamchatnov et al. (2012), and it emerges that there are sixteen different cases to be considered. Although these Whitham modulation equations could be readily extended to allow for variable coefficients in the same manner as was done here, the detailed analysis and necessary numerical simulations will be a topic for future study.

\section{References}

Ablowitz, M. J. and Segur, H. (1981). Solitons and the Inverse Scattering Transform. SIAM, Philadelphia.

Benjamin, T. B. (1966). Internal waves of finite amplitude and permanent form. J. Fluid Mech, 25:241-270.

Benney, D. J. (1966). Long non-linear waves in fluid flows. J. Math. Phys, 45:52-63.

Boyd, J. P. (2001). Chebyshev and Fourier spectral methods. Courier Corporation.

Cox, S. and Matthews, P. (2002). Exponential time differencing for stiff systems. Journal of Computational Physics, 176(2):430-455.

El, G. (2007). Kortweg-de Vries equation and undular bores. In Grimshaw, R., editor, Solitary waves in fluids, pages 19-53. Advances in Fluid Mechanics, 47, WIT Press.

El, G., R.H.J., G., and Tiong, W. (2012). Transformation of a shoaling undular bore. J. Fluid Mech., 709:371-395.

Fornberg, B. and Whitham, G. B. (1978). A numerical and theoretical study of certain nonlinear wave phenomena. Phil.Trans. Royal Soc. A, 289:373-404.

Grimshaw, R. (1979). Slowly varying solitary waves i. Korteweg-de Vries equation. Proc. Roy. Soc, 368A:359-375.

Grimshaw, R. (1981). Evolution equations for long nonlinear internal waves in stratified shear flows. Stud. Appl. Math, 65:159-188. 
Grimshaw, R. (2001). Internal solitary waves. In Grimshaw, R., editor, Environmental Stratified Flows, pages 1-27. Kluwer, Boston.

Grimshaw, R. (2007). Internal solitary waves in a variable medium. Gesellschaft fur Angewandte Mathematik, 30:96-109.

Grimshaw, R. (2015). Change of polarity for periodic waves in the variable-coefficient Kortewegde Vries equation. Stud. Appl. Math., 134:363-371.

Grimshaw, R., Pelinovsky, E., and Talipova, T. (2007). Modeling internal solitary waves in the coastal ocean. Surveys in Geophysics, 28:273-298.

Grimshaw, R., Pelinovsky, E., Talipova, T., and Kurkina, A. (2010). Internal solitary waves: propagation, deformation and disintegration. Nonlinear Processes in Geophysics, 17:633-649.

Gurevich, A. V. and Pitaevskii, L. P. (1974). Nonstationary structure of a collisionless shock wave. Sov. Phys. JETP, 38:291-297.

Helfrich, K. R. and Melville, W. K. (2006). Long nonlinear internal waves. Ann. Rev. Fluid Mechanics, 38:395-425.

Holloway, P., Pelinovsky, E., and Talipova, T. (2001). Internal tide transformation and oceanic internal solitary waves. In Grimshaw, R., editor, Environmental Stratified Flows, pages 31-60. Kluwer, Boston.

Kamchatnov, A. M. (2000). Nonlinear periodic waves and their modulations. An introductory course. World Scientific.

Kamchatnov, A. M. (2004). On Whitham theory for perturbed integrable equations. Physica $D, 188: 247-261$.

Kamchatnov, A. M., Kuo, Y.-H., Lin, T.-C., Horng, T.-L., Gou, S.-C., Clift, R., El, G., and Grimshaw, R. (2012). Undular bore theory for the Gardner equation. Phys.Rev. E, 86:036605.

Kassam, A.-K. and Trefethen, L. N. (2005). Fourth-order time-stepping for stiff pdes. SIAM Journal on Scientific Computing, 26(4):1214-1233.

Myint, S. and Grimshaw, R. (1995). The modulation of nonlinear periodic wavetrains by dissipative terms in the Korteweg-de Vries equation. Wave Motion, 22:215-238.

Ostrovsky, L. A. and Stepanyants, Y. A. (2005). Internal solitons in laboratory experiments: Comparison with theoretical models. Chaos, 28:037111.

Vlasenko, V. I., Stashchuk, N. M., and Hutter, K. (2005). Baroclinic Tides: Theoretical Modelling and Observational Evidence. Cambridge University Press.

Weideman, J. A. and Reddy, S. C. (2000). A matlab differentiation matrix suite. ACM Transactions on Mathematical Software (TOMS), 26(4):465-519.

Whitham, G. B. (1965). Non-linear dispersive waves. Proc. Roy. Soc. London A, 283:238-261.

Whitham, G. B. (1974). Linear and Nonlinear Waves. J. Wiley and Sons. 
Zhou, X. and Grimshaw, R. (1989). The effect of variable currents on internal solitary waves. Dyn. Atmos. Oceans, 14:17-39. 



Figure 5: A simulation of the vKdV equation (12) when $\alpha(\tau)$ varies from -1 to 1 as specified by (35) for the undular bore initial condition (38) with $U_{0}=-12$ and $K=5, \tau_{0}=4$; the top panel is at $\alpha=-1$, the middle panel is at $\alpha=0$ and the bottom panel is at $\alpha=1$. Note the slight variation in the horizontal and vertical axes. 



Figure 6: A simulation of the vKdV equation (12) when $\alpha=-1$ is constant, for the undular bore initial condition (38) with $U_{0}=-12$ and $\tau_{0}=4$; the top panel, middle panel and the bottom panel are at the same times as the corresponding panels in figure 5 . Note the slight variation in the horizontal and vertical axes. 

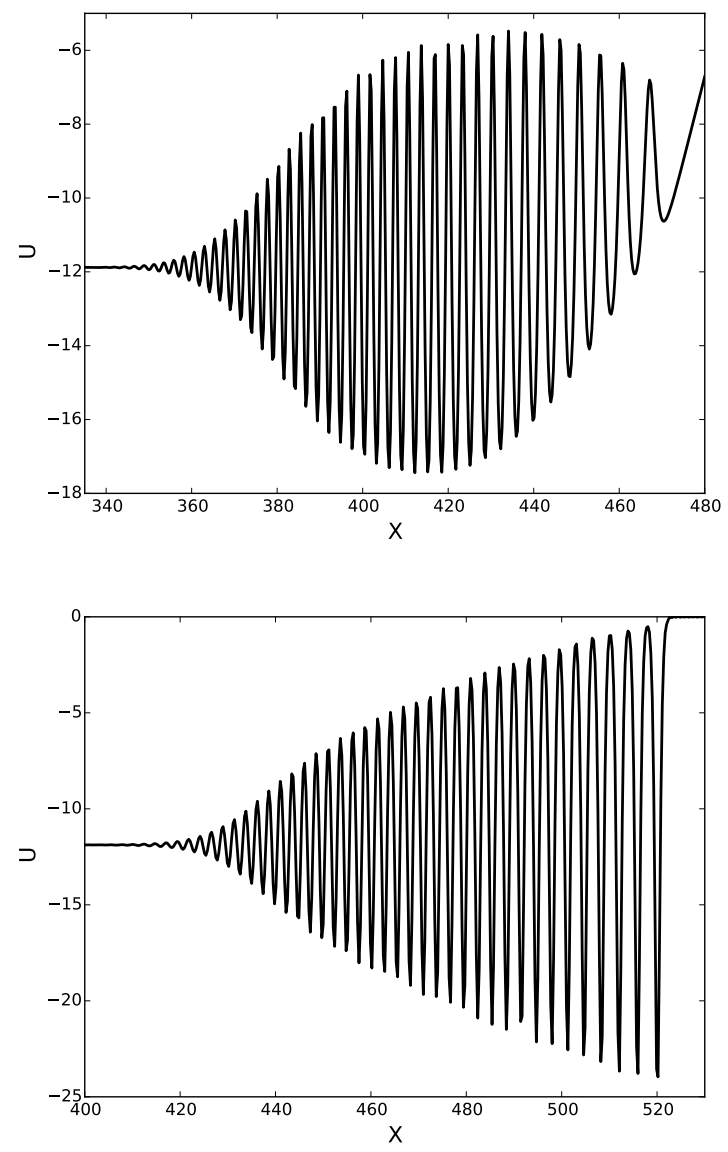

Figure 7: A simulation of the $v K d V$ equation (12) for the undular bore initial condition (38) with $U_{0}=-12$ and $K=1, \tau_{0}=5$; Both panels are at the final stage; the top panel is when $\alpha(\tau)$ varies from -1 to 1 and shows the outcome when $\alpha=1$; the bottom panel is when $\alpha=-1$ is constant. Note the slight variation in the horizontal and vertical axes. 